\title{
Cholecystectomy by a combined transgastric and transparietal approach using two flexible endoscopes
}

\author{
Nobutsugu Abe $\cdot$ Hirohisa Takeuchi $\cdot$ Hisayo Ueki $\cdot$ \\ Hiroyoshi Matsuoka - Osamu Yanagida - Tadahiko Masaki · \\ Toshiyuki Mori $\cdot$ Masanori Sugiyama $\cdot$ Yutaka Atomi
}

Received: 22 February 2008/Accepted: 24 March 2008/Published online: 17 December 2008

(C) Springer 2008

\begin{abstract}
Objective This experimental study was designed to assess the technical feasibility and benefits of our novel approach for transgastric NOTES (natural orifice translumenal endoscopic surgery) cholecystectomy.

Methods Four pigs were subjected to NOTES cholecystectomy by the combined transgastric and transparietal approach using two flexible endoscopes. Under the guidance of a transparietal endoscope inserted through a trocar placed in the right upper abdomen, a gastrotomy was constructed, and a peroral endoscope was advanced into the peritoneal cavity through the gastrotomy and moved on retroflexion toward the gallbladder. Gallbladder excision with ligation of the cystic artery and duct using endoclips was performed using the peroral endoscope. After gastrotomy closure with endoclips inside the stomach, intraperitoneal lavage were carried out using the transparietal endoscope.

Results A complete gallbladder excision was carried out without major adverse events in all cases. The gastrotomies were successfully closed using endoclips $(n=3)$ or by the omentum-plug method $(n=1)$.

Conclusion This approach is technically feasible and makes transgastric NOTES cholecystectomy easier and safer.
\end{abstract}

Keywords Natural orifice translumenal endoscopic surgery · NOTES · Transgastric cholecystectomy

N. Abe $(\bowtie) \cdot$ H. Takeuchi $\cdot$ H. Ueki $\cdot$ H. Matsuoka

O. Yanagida - T. Masaki - T. Mori - M. Sugiyama · Y. Atomi

Department of Surgery, Kyorin University School of Medicine,

6-20-2 Shinkawa, Mitaka, Tokyo 181-8611, Japan

e-mail: abenbtg@kyorin-u.ac.jp

\section{Introduction}

Natural orifice translumenal endoscopic surgery (NOTES) has been proposed as a less invasive alternative to a conventional open or laparoscopic surgery [1]. The most important advantage of NOTES is zero or minimal trauma to the abdominal wall; therefore, it is expected to reduce postoperative pain, wound complications, and the length of hospital stay. Many reports on a wide variety of NOTES procedures performed via transgastric [1-10] transcolonic [11], transvesicle [10, 12], and transvaginal [13-15] access have been published. A group at the New York Presbyterian/Columbia University Medical Center in New York City carried out the first successful laparoscopically assisted transvaginal cholecystectomy in a human patient. Marescaux et al. [15] have also demonstrated that transvaginal cholecystectomy under laparoscopic guidance with a 2-mm needle scope can be performed in a patient, with good results.

The transgastric approach seems to be the predominant route in NOTES in the future [15]; however, a number of critical problems still need to be solved [8-11], including limitations in spatial orientation in the peritoneal cavity $[8$, 9]. In particular, transgastric-only cholecystectomy is complicated because the surgeon must work with a flexible endoscope in a retroflexed position, which leads to spatial incongruity and orientation difficulties [9]. Challenges in the form of gallbladder identification and cystic pedicle exposure have been reported with the exclusive use of a transgastric port $[2,9,11]$. A second problem is controlling potential infection of the intraperitoneal surgical site [9]. During surgery, the spillage of gastric juice through the gastrotomy is inevitable, and bile spillage may occasionally occur. The potential remnant of gastric contents and/or bile can be an infection source. A third problem is whether 
the gastrotomy can be closed completely. The existence of such unresolved problems is the reason why transgastriconly NOTES cholecystectomy has not yet been introduced in clinical settings.

We hypothesized that the combined transgastric and transparietal approach using two flexible endoscopes can circumvent such problems associated with transgastriconly NOTES cholecystectomy. The experimental study reported here was performed to determine whether this combined approach for NOTES cholecystectomy can overcome the problems associated with transgastric-only cholecystectomy. The main goal of this study was to assess the technical feasibility and benefits of this combined approach.

\section{Materials and methods}

Animals and equipment

Natural orifice translumenal endoscopic surgery cholecystectomy by the combined transgastric and transparietal approach using two flexible endoscopes was attempted in four nonsurvival experiments on pigs (body weight $30-40 \mathrm{~kg}$ ). The ethical review board of our institution approved the protocol for this study. The pigs were fasted for $40 \mathrm{~h}$ before the experiments. The procedures were carried out under general endotracheal anesthesia. Following the surgery, the animals were euthanized, and necropsy was carried out. The intraperitoneal organs, resection bed, cystic stump, gallbladder specimen, and gastrotomy site were thoroughly examined.

Two flexible, forward-viewing endoscopes (GIF-Q260 J: external diameter $9.9 \mathrm{~mm}$, full length $1.35 \mathrm{~m}$; Olympus Medical Systems, Tokyo, Japan) with a water-jet system and commercially available flexible endoscopic accessories were used. The endoscopic accessories included a needle knife (KD-1L-1; Olympus), a 16-mm-diameter dilation balloon (ECL-16 × 5.5; Wilson-Cook Medical, Winston-Salem, NC), a Hook knife (KD-620LR; Olympus), a Flex knife (KD-630L; Olympus), a rotatable clip-fixing device (HX-110QR; Olympus), endoclips (HX-610-135, HX-610-135L; Olympus), and a grasping forceps (FG-47L-1; Olympus). Two videoprocessors and monitors were used to acquire and display the images from both endoscopes. An endosurgical generator (PSD-60; Olympus) was used for incision, dissection, and electrocoagulation during surgery. A 12-mm trocar (a device for laparoscopic surgery; Endopath Xcel; Ethicon Endo-Surgery, Cincinnati, $\mathrm{OH}$ ), and a grasping forceps (a device for laparoscopic surgery; A63073A; Olympus) were also used.
Surgical techniques

Two of the operators who carried out the combined procedures were gastroenterological surgeons well-experienced in both laparoscopic cholecystectomy and advanced therapeutic endoscopy, such as endoscopic submucosal dissection [16, 17].

A 12-mm initial access site was established in the right upper quadrant of the abdominal wall by the Hasson open technique. A 12-mm trocar was introduced into the peritoneal cavity, and a pneumoperitoneum was generated with carbon dioxide at a pressure of $10-15 \mathrm{mmHg}$ using a standard laparoscopic insufflator (UHI-3; Olympus), followed by the insertion of an endoscope (transparietal endoscope) through the trocar into the peritoneal cavity.

An overtube was introduced perorally into the esophagus, and an endoscope (peroral endoscope) was advanced through the overtube into the stomach. Under direct transparietal endoscopic vision, a 2- to 3-mm full-thickness incision of the stomach was made using a needle knife in the anterior wall of the lower body or the antrum of the stomach and dilated to $16 \mathrm{~mm}$ using a balloon dilator. The peroral endoscope was then advanced into the peritoneal cavity through the gastrotomy. The transparietal endoscope was first used to observe the position of the peroral endoscope and to facilitate its orientation in the peritoneal cavity (Fig. 1a). The peroral endoscope was moved on retroflexion toward the gallbladder (Fig. 1a). Once the gallbladder was identified through the peroral endoscope, the parietal endoscope was removed, and then a laparoscopic grasping forceps was inserted through the trocar into the peritoneal cavity. Under the guidance of the peroral endoscope, the gallbladder fundus was grasped using the forceps and tracted toward the right diaphragm and/or to the anterior abdominal wall for the necessary exposure of the cystic artery and duct (Fig. 1b). The dissection was begun in close proximity to the gallbladder, at the junction between the infundibulum and cystic artery and duct. After both sides of the cystic artery and duct were dissected, they were clipped twice on the bile duct side and once on the gallbladder side and divided using a Hook knife. The gallbladder was then dissected from the intrahepatic fossa using the Hook knife or the Flex knife and retrieved by withdrawing the peroral endoscope into the stomach and through the overtube. The gastrotomy was closed inside the stomach lumen using endoclips. To confirm the completeness of the closure of the gastrotomy, after the stomach lumen was insufflated, air leakage was checked under direct vision through the transparietal endoscope. After the closure of the gastrotomy, the surgical site in the peritoneal cavity was lavaged with $500 \mathrm{~mL}$ of saline using the transparietal endoscope and, finally, the intraperitoneal fluid was aspirated. 


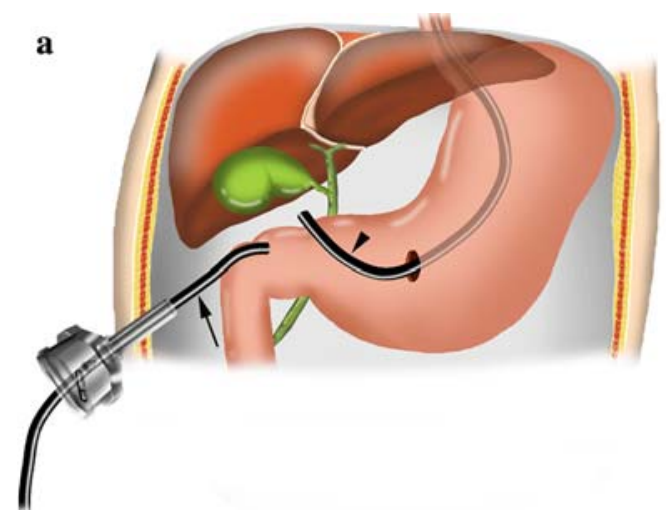

Fig. 1 Schematic of natural orifice translumenal endoscopic surgery (NOTES) cholecystectomy by combined transgastric and transparietal approach using two flexible endoscopes. a The transparietal endoscope (arrow) is first used to observe the position of the peroral

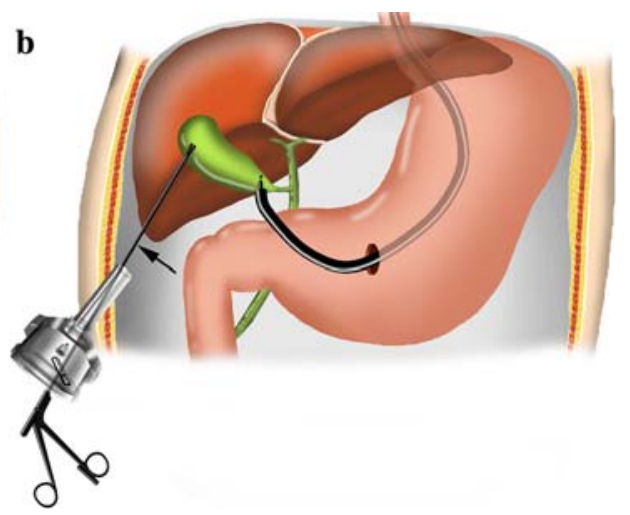

endoscope (arrowhead) and to facilitate its orientation in the peritoneal cavity. b The gallbladder traction using a laparoscopic grasping forceps (arrow) inserted through the trocar provides excellent visualization and access to the gallbladder
Fig. 2 Transparietal

endoscopic view. a Under direct transparietal endoscopic vision, the small full-thickness incision made using a needle knife is dilated to $16 \mathrm{~mm}$ using a balloon dilator. b Under direct transparietal endoscopic vision, the peroral endoscope is moved on retroflexion toward the gallbladder. This navigation enables easy positioning of the peroral endoscope after passing through the gastrotomy
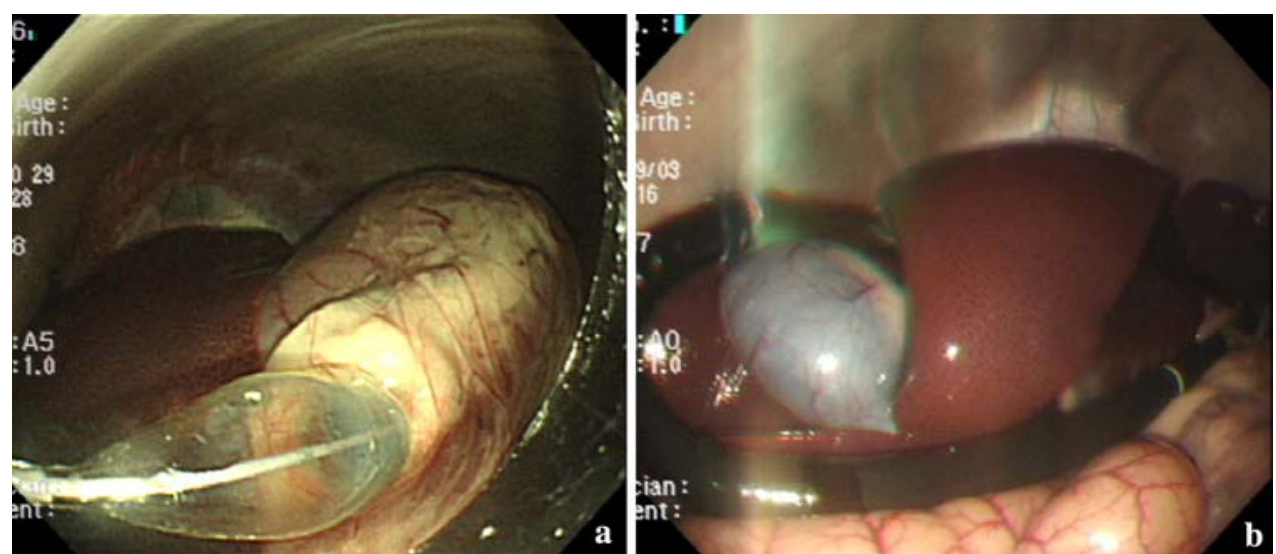

\section{Results}

The transparietal endoscope provided a perfect view of the intraperitoneal cavity and enabled the easy identification of the gallbladder and the stomach. The gastric incision and balloon dilatation (gastrotomy construction) were carried out under direct vision through the transparietal endoscope without incurring significant damages to the adjacent structures (Fig. 2a). The transparietal endoscopic view was excellent for the navigation of the peroral endoscope in the peritoneal cavity-namely, the peroral endoscope enabled easy identification of the gallbladder without any difficulties after passing through the gastrotomy (Fig. 2b).

The grasping and tracting of the transparietal gallbladder allowed the peroral endoscope operator to quickly identify the cystic artery and duct. The gallbladder traction provided excellent visualization and access to the cystic artery and duct (Fig. 3a). A complete gallbladder excision, with ligation of the cystic vessels and duct using endoclips (Fig. 3b), was completed in all cases. During the detachment of the gallbladder from the liver bed (Fig. 3c), a minor perforation (1 $\mathrm{mm}$ in diameter) of the gallbladder occurred in two cases during the use of the tip of the Hook knife; however, in both cases, no significant bile leakage occurred. Liver-surface bleeding occurred on occasion during the course of the dissection. This either stopped spontaneously or was easily managed by an electrocoagulation using the cutting knife (the Hook knife or Flex knife).

The gastrotomy was successfully closed using five to eight endoclips from inside the stomach lumen in three of the four cases (Fig. 4a). It was not possible to close the gastrotomy with endoclips owing to its size and shape (diameter $15 \mathrm{~mm}$, round-shaped) in one case. In this case, an omentum-plug method [18] was carried out using the greater omentum as a plug. The omentum was grasped by a grasping forceps outside the gastrotomy and was brought into the gastric lumen, following which the omentum and mucosa around the gastrotomy were fixed using several endoclips (Fig. 4b). During this procedure, from outside the stomach, the omentum was pushed to the gastrotomy site using the grasping forceps through the transparietal 
Fig. 3 Peroral (transgastric) endoscopic view. a Under peroral endoscopic vision, the gallbladder fundus was grasped using the forceps and tracted for the exposure of the cystic artery and duct; arrow trocar placed in right upper abdomen. b Division of cystic artery and duct clipped using the Hook knife (arrow). c Dissection of gallbladder from intrahepatic fossa using the Hook knife (arrow). Arrowhead Transparietal grasping forceps, $G B$ gallbladder

Fig. 4 Gastrotomy closure and intraperitoneal lavage. a Peroral endoscopic view after the simple closure of the gastrotomy using endoclips. b Peroral endoscopic view of the omental-plug for the gastrotomy closure. The omentum (arrowheads) brought into the inner aspect of the stomach was fixed using several endoclips. c Transparietal endoscopic view of the closure site (arrow) of the gastrotomy. Air leakage was not observed even after the stomach lumen was fully insufflated. d Transparietal endoscopic view of the intraperitoneal lavage using the transparietal endoscope after the gastrotomy closure; arrowheads saline discharged from the endoscope
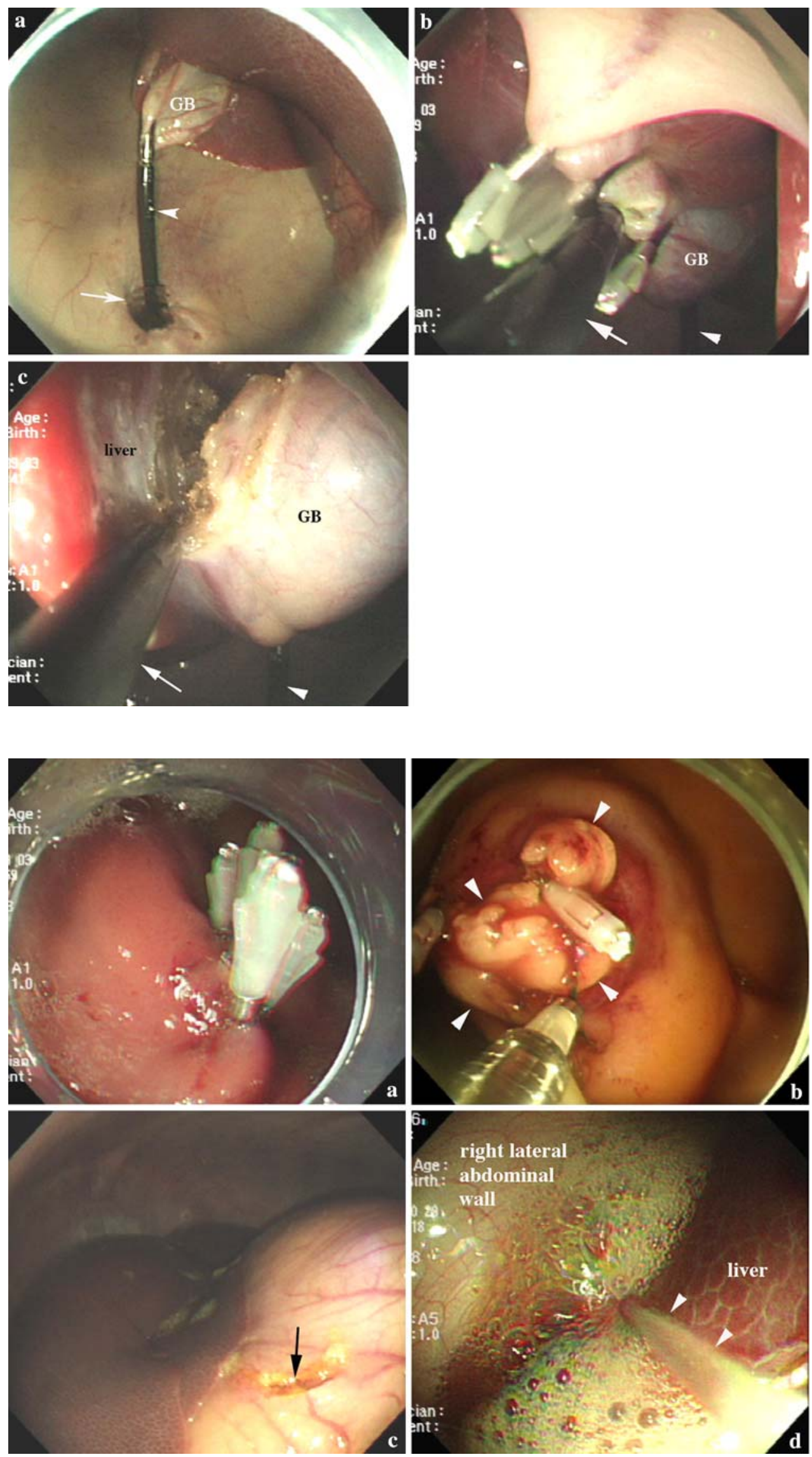
endoscope. In all cases, the completeness of the closure of the gastrotomy was confirmed by direct visualization through the transparietal endoscope from inside the peritoneal cavity (Fig. 4c). Air leakage was not observed even after the stomach was fully insufflated.

The peritoneal lavage using the transparietal endoscope after the gastrotomy closure was successfully carried out in all cases (Fig. 4d). The aspiration of the intraperitoneal fluid through the endoscope was satisfactory. The mean operating time was $200 \mathrm{~min}$ (range 171-243 min).

Postmortem examination revealed that the endoclips secured the cystic artery and duct and that neither bile nor blood leaked from this site. The fluid remnant in the peritoneal cavity was minimum. The gastrotomy was completely closed using endoclips in three cases or an omentum-plug in one case.

\section{Discussion}

The results of our experimental study clearly demonstrated that this approach (NOTES cholecystectomy by the combined transgastric and transparietal approach using two flexible endoscopes) addresses the critical problems associated with transgastric-only cholecystectomy. The transparietal endoscopic vision allows for the appropriate positioning of the gastric incision site, a safe construction of the gastrotomy without the incurrence of any damage to the adjacent structures, a good navigation for the peroral endoscope in the peritoneal cavity, and the confirmation of gastrotomy closure. The transparietal endoscope can also facilitate gastrotomy closure from outside the stomach and additionally allows for intraperitoneal lavage. Furthermore, a trocar placed in the abdominal wall for the insertion of the transparietal endoscope can be mandatory to insufflate carbon dioxide and to monitor for pneumoperitoneum, and can be used as an access port for a grasping forceps for gallbladder traction. The gallbladder traction using the forceps is essential for the safe use of the peroral endoscope. Thus, this approach has many advantages over the previously reported transgastric-only cholecystectomy [2].

Other authors $[8,9]$ have also recently reported the successful use of a transparietal laparoscope to assist transgastric NOTES cholecystectomy in procedures almost the same as the one reported here. Such "hybrid techniques" $[8,9]$ appear to be successful in overcoming the limitation of spatial orientation in the peritoneal cavity. However, there are some notable differences between our technique (the use of a flexible endoscope) and those of others (the use of a rigid laparoscope) [8, 9]. The most important advantages of our technique over the "hybrid techniques" [8,9] are: (1) the transparietal endoscope can facilitate gastrotomy closure from outside the stomach; (2) this technique can allow for intraperitoneal lavage. A laparoscope that includes a working channel which allows for the passage of large instruments is not yet commercially available. Consequently, there is as yet no laparoscope available that can assist in the work of gastrotomy closure from outside the stomach and which can provide intraperitoneal lavage and aspiration-in contrast with our technique. A rigid 5-mm ureterosope that includes a working channel [12] might be replaced by the flexible endoscope. However, we consider that this scope would not be adequate for satisfactory intraperitoneal lavage and aspiration owing to diameter limitation.

The spillage of gastric contents through the gastrotomy is inevitable during transgastric NOTES. Although gastric lavage with an antibiotic solution prior to the gastric incision, together with systemic antibiotic treatment, has reportedly been an effective prophylactic measure against postoperative infection in animal models [3], a near-zero postoperative infection rate should be assured to justify the use of transgastric NOTES in humans. At the present time, the potential intraperitoneal infection is likely the most important issue limiting the widespread use of transgastric NOTES in humans. For this reason, we consider that intraperitoneal lavage after the gastrotomy closure is an essential step in transgastric NOTES. Intraperitoneal lavage can remove the gastric contents, the occasional presence of bile, and contaminated contents via the peroral transgastric endoscope. Thus, this procedure may minimize or completely eliminate the risk of postoperative infection. On the basis of the above considerations, we used a flexible endoscope as an adjunct scope. A laparoscope with a water-jet and aspiration system that includes a large working channel (currently not available) may eventually be useful for our purpose.

The urinary bladder has also been described as an entry port for peritoneoscopy [10, 12]. Rolanda et al. [10] have recently demonstrated that the combined transgastric and transvesicle approach for NOTES cholecystectomy can be used to overcome several problems associated with transgastric-only cholecystectomy. From the viewpoint of the NOTES concept-i.e., incisionless or no-scar operationRolanda's approach appears to be "true" NOTES cholecystectomy. However, we must be concerned with the fact that two iatrogenic injuries of intraperitoneal organs (stomach and urinary bladder) as entry ports are required for this approach. It is unclear if such approach is really acceptable in humans. In contrast, our approach consists of a single iatrogenic injury of an intraperitoneal organ (stomach) and a single abdominal incision. We believe that our approach, one of the combinations of NOTES and endoscopic surgery, is more widely acceptable than that of Rolanda et al. 
The acute experimental study reported here was only designed to assess the technical feasibility and benefits of our novel approach for transgastric NOTES cholecystectomy. Although it will require many experiments on animals and survival studies before the true utility of this new approach is established, our approach may represent possible routes for minimally invasive surgery in the abdomen.

In conclusion, the approach described here is technically feasible and makes transgastric NOTES cholecystectomy easier and safer. It may circumvent the major problems associated with transgastric-only NOTES cholecystectomy.

\section{References}

1. Kalloo AN, Singh VK, Jagannath SB, Niiyama H, Hill SL, Vaughn CA, et al. Flexible transgastric peritoneoscopy: a novel approach to diagnostic and therapeutic interventions in the peritoneal cavity. Gastrointest Endosc. 2004;60:114-7.

2. Park PO, Bergström M, Ikeda K, Fritscher-Ravens A, Swain P. Experimental studies of transgastric gallbladder surgery: cholecystectomy and cholecystogastric anastomosis (videos). Gastrointest Endosc. 2005;61:601-6.

3. Jagannath SB, Kantsevoy SV, Vaughn CA, Chung SS, Cotton $\mathrm{PB}$, Gostout CJ, et al. Peroral transgastric endoscopic ligation of fallopian tubes with long-term survival in a porcine model. Gastrointest Endosc. 2005;61:449-53.

4. Kantsevoy SV, Hu B, Jagannath SB, Vaughn CA, Beitler DM, Chung SS, et al. Transgastric endoscopic splenectomy: is it possible? Surg Endosc. 2006;20:522-25.

5. Merrifield BF, Wagh MS, Thompson CC. Peroral transgastric organ resection: a feasibility study in pigs. Gastrointest Endosc. 2006;63:693-707.

6. Sumiyama K, Gostout CJ, Rajan E, Bakken TA, Deters JL, Knipschield MA, et al. Pilot study of the porcine uterine horn as an in vivo appendicitis model for development of endoscopic transgastric appendectomy. Gastrointest Endosc. 2006;64:80812 .

7. Kantsevoy SV, Jagannath SB, Niiyama H, Isakovich NV, Chung $\mathrm{SS}$, Cotton PB, et al. A novel safe approach to the peritoneal cavity for per-oral transgastric endoscopic procedures. Gastrointest Endosc. 2007;65:111-117.

8. Mintz Y, Horgan S, Cullen J, Ramamoorthy S, Chock A, Savu MK, et al. NOTES: the hybrid technique. J Laparoendosc Adv Surg Tech A. 2007;17:402-406.

9. Shih SP, Kantsevoy SV, Kalloo AN, Magano P, Giday SA, Ko $\mathrm{CW}$, et al. Hybrid minimally invasive surgery—a bridge between laparoscopic and translumenal surgery. Surg Endosc. 2007;21:1450-53.

10. Rolanda C, Lima E, Pêgo JM, Henriques-Coelho T, Silva D, Osório L, et al. Third-generation cholecystectomy by natural orifices: transgastric and transvesical combined approach (with video). Gastrointest Endosc. 2007;65:111-7.

11. Pai RD, Fong DG, Bundga ME, Odze RD, Rattner DW, Thompson CC. Transcolonic endoscopic cholecystectomy: a NOTES survival study in a porcine model (with video). Gastrointest Endosc. 2006;64:428-31.

12. Lima E, Rolanda C, Pêgo JM, Henriques-Coelho T, Silva D, Carvalho JL, et al. Transvesicle endoscopic peritoneoscopy: a novel 5-mm port for intra-abdominal scarless surgery. J Urol. 2006; 176:802-5.

13. Gettman MT, Lotan Y, Napper CA, Cadeddu JA. Transvaginal laparoscopic nephrectomy: development and feasibility in the porcine model. Urology. 2002;59:446-50.

14. Scott DJ, Tang SJ, Fernandez R, Bergs R, Goova MT, Zeltser I, et al. Completely transvaginal NOTES cholecystectomy using magnetically anchored instruments. Surg Endosc. 2007;21:230816.

15. Marescaux J, Dallemagne B, Perretta S, Wattiez A, Mutter D, Coumaros D. Surgery without scars: report of transluminal cholecystectomy in a human being. Arch Surg. 2007;142:823-6.

16. Abe N, Mori T, Takeuchi H, Yoshida T, Ohki A, Ueki H, et al. Laparoscopic lymph node dissection after endoscopic submucosal dissection: a novel and minimally invasive approach to treating early-stage gastric cancer. Am J Surg. 2005;190:496503.

17. Abe N, Yamaguchi Y, Takeuchi H, Izumisato Y, Yanagida O, Masaki T, et al. Key factors for successful en bloc endoscopic submucosal dissection of early stage gastric cancer using an insulation-tipped diathermic knife. Hepatogastroenterology. 2006;53:639-42.

18. Minami S, Gotoda T, Ono H, Oda I, Hamanaka H. Complete endoscopic closure of gastric perforation induced by endoscopic resection of early gastric cancer using endoclips can prevent surgery (with video). Gastrointest Endosc. 2006;63:596-601. 